\title{
Unrecognized liver injury assessed by fibrosis indexes is associated with mortality in critically ill COVID-19 patients
}

\author{
Mario Romero-Cristobal \\ HGU. Gregorio Marañón
}

Ana Clemente

HGU. Gregorio Marañón

Patricia Piñeiro

HGU Gregorio Marañón

Jamil Cedeño

HGU. Gregorio Marañón

Laura Rayón

HGU. Gregorio Marañón

Julia Del Río

HGU. Gregorio Marañón

Clara Ramos

HGU. Gregorio Marañón

Diego-Andrés Hernández

HGU. Gregorio Marañón

Miguel Cova

HGU. Gregorio Marañón

Aranzazu Caballero

HGU. Gregorio Marañón

Ignacio Garutti

HGU. Gregorio Marañón

Pablo García-Olivares

HGU. Gregorio Marañón

Javier Hortal

HGU. Gregorio Marañón

Jose-Eugenio Guerrero

HGU. Gregorio Marañón

\section{Rita García}

Instituto de Salud Carlos III

Rafael Bañares ( $\nabla$ rbanares@ucm.es) 
HGU. Gregorio Marañón, Madrid

\section{Diego Rincón}

HGU. Gregorio Marañón

\section{Research Article}

Keywords: coronavirus, liver diseases, biomarkers, critical care, survival analysis, liver cirrhosis, comorbidity, regression analysis, APACHE, prognosis

Posted Date: September 29th, 2020

DOl: https://doi.org/10.21203/rs.3.rs-83678/v1

License: (9) This work is licensed under a Creative Commons Attribution 4.0 International License. Read Full License 
Manuscript under review. Hepatology International (HEPI-D-20-00795)

Unrecognized liver injury assessed by fibrosis indexes is associated with mortality in critically ill COVID-19 patients.

Mario Romero-Cristóbal (1), Ana Clemente-Sánchez (1,6), Patricia Piñeiro

(2), Jamil Cedeño (3), Laura Rayón (1), Julia del Río (1), Clara Ramos (1),

Diego-Andrés Hernández (1), Miguel Cova (1), Aranzazu Caballero (1), Ignacio Garutti (2), Pablo García-Olivares (3), Javier Hortal (2,4,5), Jose-Eugenio Guerrero (3), Rita García (6,7), Rafael Bañares (1,5,6), Diego Rincón $(\mathbf{1 , 5 , 6 )}$.

1.-Liver Unit and Digestive Department H.G.U. Gregorio Marañón, Madrid. Spain.

2.- Department of Anesthesiology H.G.U. Gregorio Marañón, Madrid. Spain.

3.- Intensive Care Unit H.G.U. Gregorio Marañón, Madrid. Spain.

4.- CIBERES. Instituto de Salud Carlos III. Madrid

5.- School of Medicine, Complutense University, Madrid. Spain.

6.- CIBEREHD. Instituto de Salud Carlos III. Madrid

7.- Department of Internal Medicine H.G.U. Gregorio Marañón, Madrid. Spain. 
E-mail address:

mario romero cristobal@hotmail.es

anacs@pitt.edu

patricia.pineiro@salud.madrid.org

jamilantonio.cedeno@salud.madrid.org

I.rayon.Ir@gmail.com

juliadelrioizquierdo@gmail.com

clararamosbel@gmail.com

diego.hernandez.castillo@gmail.com

miguel.cova@salud.madrid.org

aranzazu.caballero@salud.madrid.org

ignacio.garutti@salud.madrid.org

pgolivares@salud.madrid.org

franciscojavier.hortal@salud.madrid.org

joseeugenio.guerrero@salud.madrid.org

humrita77@yahoo.es

drinco01@ucm.es 
Mario Romero-Cristóbal and Ana Clemente-Sánchez share first authorship

Rafael Bañares and Diego Rincón share senior authorship.

Corresponding author:

Rafael Bañares Cañizares.

Hospital General Universitario Gregorio Marañón. Liver Unit.

Doctor Esquerdo 46. 28007. Madrid.

Phone Number: +34915868000

Email: rbanares@ucm.es

Compliance with ethical requirements: all the authors declare that they have no conflict of interest. The study was approved by the local Ethics Committee (May $11^{\text {th }}$ 2020).

\section{Abbreviations.}


ACEI: angiotensin converting enzyme inhibitors.

ALT: alanine aminotransferase

APACHE II: Classification System for Chronic Diseases and Acute Health Physiology II.

ARB: angiotensin receptor blockers.

ARDS: acute respiratory distress syndrome.

AST: aspartate aminotransferase.

CCI: Charlson Comorbidity Index.

COPD: chronic obstructive pulmonary disease.

COVID-19: coronavirus disease 2019.

ICU: intensive care unit.

MAFLD: metabolic dysfunction-associated fatty liver disease.

NAFLD: non alcoholic fatty liver disease.

NFS: NAFLD fibrosis score.

ROC: receiver operator characteristics curve.

RT-PCR: reverse-transcriptase-polymerase-chain-reaction.

\section{Authors contributions:}


Conceptualization: Mario Romero-Cristóbal, Ana Clemente-Sánchez, Rita García, Rafael Bañares, Diego Rincón.

Acquisition of data: Patricia Piñeiro, Jamil Cedeño, Laura Rayón, Julia del Río, Clara Ramos, Diego-Andrés Hernández, Miguel Cova, Aranzazu Caballero, Ignacio Garutti, Pablo García-Olivares, Francisco-Javier Hortal, Jose-Eugenio Guerrero.

Formal analysis and methodology: Mario Romero-Cristóbal, Ana ClementeSánchez, Rita García, Rafael Bañares, Diego Rincón.

Proyect administration: Diego Rincón.

Writing - original draft: Mario Romero-Cristóbal, Ana Clemente-Sánchez, Rita García, Rafael Bañares, Diego Rincón.

Writing - review and editing: Mario Romero-Cristóbal, Ana Clemente-Sánchez, Rita García, Rafael Bañares, Diego Rincón.

Figure legend.

Figure 1. Panel A Distribution of patients according to different fibrosis categories established by baseline Forns and FIB-4. Panel B Stratification of NAFLD fibrosis score values $(n=95)$ according fibrosis severity determined by Forns and FIB-4. p-value for mean comparison between categories $<0.01$ for both indexes. 
Figure 2. Kaplan - Meier survival curves according to pre-existing fibrosis. Panel A Survival curves according to the severity of fibrosis estimated by Forns index. Panel B Survival curves according to the presence or absence of fibrosis estimated by Forns Index. Panel C Survival curves according to the severity of fibrosis estimated by FIB-4 index. Panel D Survival curves according to the presence or absence of fibrosis estimated by FIB-4

Figure 3. Distribution of different liver laboratory tests in survivors and nonsurvivors at different time points during hospitalization. Kernel density estimates were used for graphical representation. p-values were non-significant in all cases. 


\begin{abstract}
Background

Coronavirus disease (COVID-19) with acute respiratory distress syndrome is a life-threatening condition. A previous diagnosis of chronic liver disease is associated with poorer outcomes. Nevertheless, the impact of silent liver injury has not been investigated. We aimed to explore the association of preadmission liver fibrosis indexes with the prognosis of critically ill COVID-19 patients.
\end{abstract}

\title{
Methods
}

Observational study in 214 patients with COVID-19 consecutively admitted to the ICU. Pre-admission liver fibrosis indexes were calculated. In-hospital mortality and predictive factors were explored with Kaplan-Meier and Cox regression analysis.

\section{$\underline{\text { Results }}$}

The mean age was 59.58 (13.79) years. Sixteen patients $(7.48 \%)$ had previously recognized chronic liver disease. Up to $78.84 \%$ of patients according to Forns, and $45.76 \%$ according to FIB-4, had more than minimal fibrosis. Fibrosis indexes were higher in non-survivors [Forns: 6.04 (1.42) vs 4.99 (1.58), $\mathrm{p}<0.001$; FIB-4: 1.77 (1.17) vs $1.41(0.91), \mathrm{p}=0.020)]$, but no differences were found in liver biochemistry parameters. Patients with any degree of fibrosis 
either by Forns or FIB-4 had a higher mortality, which increased according to the severity of fibrosis ( $p<0.05$ for both indexes). Both Forns [HR 1.41 (1.111.81); $p=0.006$ ] and FIB-4 [HR $1.31(0.99-1.72) ; p=0.051]$ were independently related to survival after adjusting for the Charlson Comorbidity Index, APACHE II and ferritin.

Conclusion

Unrecognized liver fibrosis, assessed by serological tests prior to admission, is independently associated with a higher risk of death in patients with severe COVID-19 admitted to the ICU.

Keywords: coronavirus, liver diseases, biomarkers, critical care, survival analysis, liver cirrhosis, comorbidity, regression analysis, APACHE, prognosis.

\section{Graphical abstract.}




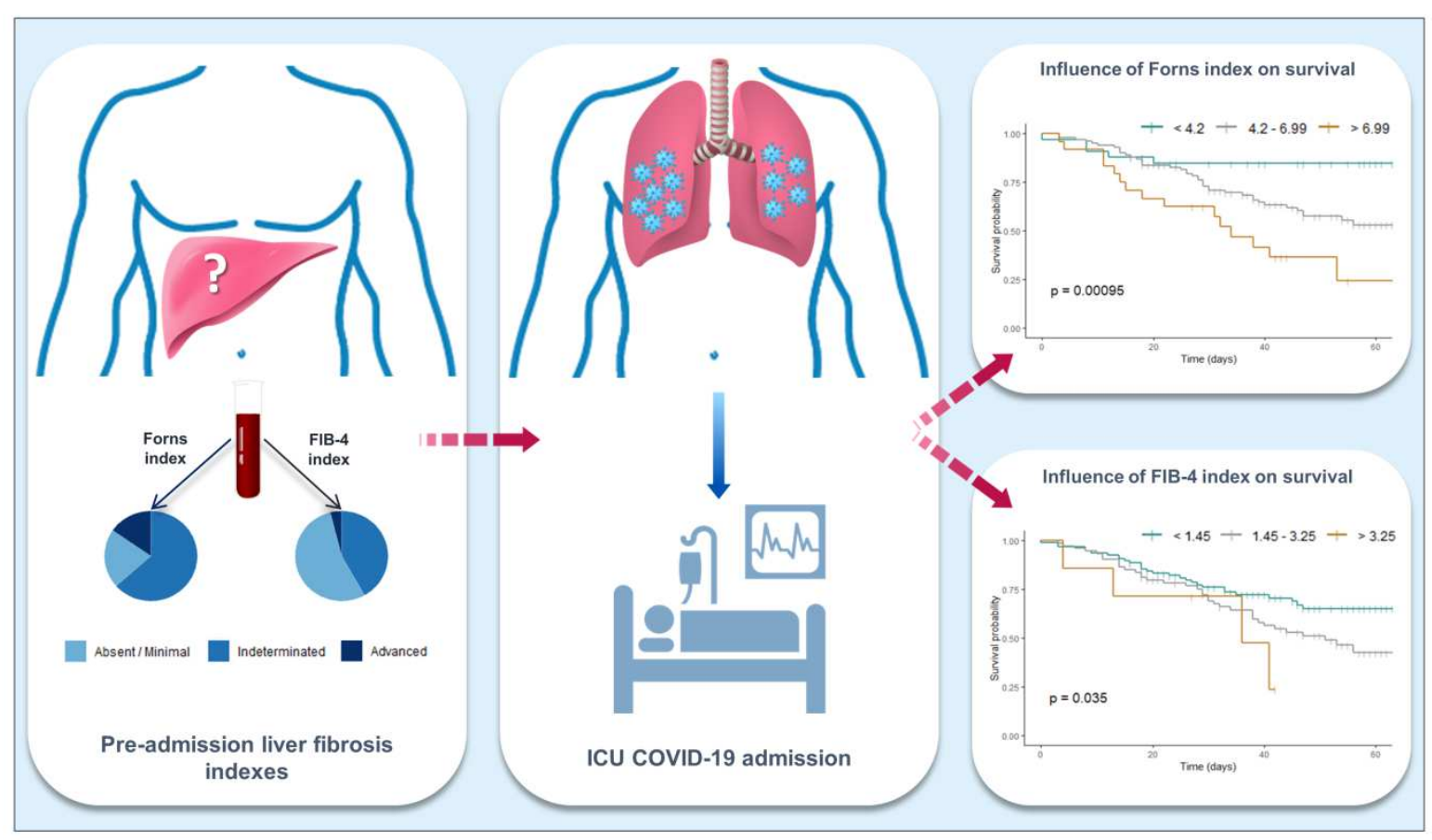

\section{INTRODUCTION}

The pandemic outbreak of the novel coronavirus (SARS-CoV-2) has become a serious public health emergency worldwide, the identification of factors associated with unfavourable outcomes being particularly important. The severity of the disease increases with older age, male gender and the presence of comorbidities, especially cardiovascular disease, hypertension, type-2 diabetes, chronic renal failure and obesity [1]. Thus, in patients hospitalized with COVID-19 and no relevant comorbidities, mortality is less than $4 \%$, while in patients suffering from 1,2 or 3 of these comorbidities, mortality increases to $14 \%, 21 \%$ and $60 \%$, respectively [2]. 
Cirrhotic patients with acute respiratory distress syndrome of any aetiology have a worse prognosis than patients without cirrhosis [3]. Preliminary data also suggest that the prognosis of COVID-19 is worse in patients with chronic liver disease, mainly in the cirrhotic stage [4-6]. Importantly, the comorbidity pattern of patients with severe COVID-19 is similar to that observed in metabolic dysfunction-associated fatty liver disease (MAFLD). In fact, a recent study has reported a higher prevalence of MAFLD in severe COVID-19 patients than in those with mild SARS-CoV-2 disease [7]. Moreover, the risk of developing severe COVID-19 in patients with MAFLD seems to be independently associated with the FIB-4 score: MAFLD patients with intermediate or high FIB-4 scores at admission, as an estimate of the existence of relevant hepatic damage, have a poorer prognosis than patients with MAFLD with a low FIB-4 score or those without MAFLD[8]. Furthermore, a recent study described the independent association between FIB-4, also obtained at the time of admission, and the need for mechanical ventilation [9]. However, there is no information about the potential impact of the presence of unrecognized liver disease on mortality in severe COVID-19 patients requiring critical care.

Therefore, the aim of the present study was to explore the possible contribution of underlying chronic liver disease, estimated by liver fibrosis indexes obtained before COVID-19 admission, to the prognosis of patients requiring intensive care management.

\section{PATIENTS AND METHODS}

\section{Study design and source of data}


This is an observational study including all patients consecutively admitted to the ICU of the Hospital General Universitario Gregorio Marañón (Madrid, Spain) with a diagnosis of COVID-19, up to April $30^{\text {th }}, 2020$. All patients had a positive result for SARS-CoV-2 on a reverse-transcriptasepolymerase-chain-reaction (RT-PCR) assay of a nasopharyngeal swab.

Demographic, clinical and laboratory results were obtained from electronic medical records and included in a database within a secure framework. Antiviral therapies during COVID-19 were also collected. Researchers analysed only de-identified data. To evaluate previous chronic comorbidities and to estimate the severity of the patient's condition at the time of ICU admission, the Charlson Comorbidity Index (CCI) [10] and the Classification System for Chronic Diseases and Acute Health Physiology II (APACHE II) score were calculated as previously described [11]. The primary study endpoint was time to in-hospital mortality.

\section{Calculation of fibrosis scores}

The FIB-4 score was calculated as age (years) ${ }^{*}$ AST (IU/L) / platelet count $\left(10^{9} / \mathrm{L}\right) * \sqrt{ } \operatorname{ALT}((\mathrm{IU} / \mathrm{L})$. FIB-4 has an area under the ROC curve of 0.85 for the prediction of significant fibrosis in chronic hepatitis $C[12,13]$ and has also been validated for the estimation of fibrosis in MAFLD [14].

The Forns index was calculated as $7.811-3.131^{\star}$ In [platelet count $\left.\left(10^{9} / \mathrm{L}\right)\right]+0.781^{*}$ In [gamma glutamyl-transferase $\left.(\mathrm{IU} / \mathrm{L})\right]+3.467^{*}$ In [age (years)] - $0.014^{*}[$ cholesterol $(\mathrm{mg} / \mathrm{dL})]$. The Forns index has an area under the ROC 
curve of 0.81 for the prediction of advanced fibrosis in chronic hepatitis $C$ [15]. As well as FIB-4, the Forns index has been accepted for the stratification of liver injury in MAFLD patients [16].

The NAFLD fibrosis score (NFS) was calculated as $0.037^{\star}$ age (years) + $0.094^{\star} \mathrm{BMI}\left(\mathrm{kg} / \mathrm{m}^{2}\right)+1.13^{*}$ [impaired fasting glucose or diabetes (yes $=1$, no $=$ $0)]+0.99^{*} \mathrm{AST}(\mathrm{IU} / \mathrm{I}) / \mathrm{ALT}(\mathrm{IU} / \mathrm{L})$ ratio $-0.013^{*}$ platelet count $\left(10^{9} / \mathrm{L}\right)$ $0.66^{\star}$ albumin $(\mathrm{g} / \mathrm{dL})-1.675$. This test was specifically developed for MAFLD patients, with an area under the ROC curve of 0.82 for the prediction of significant fibrosis [17].

All the indexes were calculated using laboratory data obtained before hospitalization for COVID-19 that were retrieved from electronic records. When more than one data set was available, we selected the one closest to admission. Only determinations obtained up to one year before COVID-19 admission were considered valid for the purpose of the study.

\section{Statistics}

Continuous variables were expressed as means (SD) or medians (IQR) as appropriate. The assumption of normality was tested by constructing normal probability plots. Categorical variables were expressed as proportions (percentage). To compare the differences in continuous variables between groups, Student's t-test, ANOVA or the Mann-Whitney $U$ test were used when appropriate. The Chi-squared or Fisher's exact tests were applied to compare categorical variables between groups.

The evolution of the different laboratory parameters during hospital stay and its relationship with survival were analysed using Kernel density estimation. 
Observed survival rates for the previously described FIB-4 and Forns index categories $[13,15]$ were calculated according to Kaplan-Meier analysis and compared with the log-rank test. Uni- and multivariate Cox regression models were developed to evaluate the independent prognostic contribution of the liver fibrosis scores. We have chosen a time-dependent multivariate model because hospital and ICU stays of patients with severe COVID-19 are often long-lasting [18]. To evaluate the independent influence of each non-invasive index on mortality, we constructed two different multivariate models that included, alternatively, FIB-4 or the Forns index. To simplify the interpretation of results and to avoid overfitting, we included in the models $\mathrm{CCl}$ (as a summary variable for age and comorbidity), APACHE II (as a standard prognostic index in ICU patients) and ferritin values (as a surrogate of the intensity of the inflammatory response). Variables that showed $p$ values equal to or lower than 0.10 in univariate analyses were included in the multivariate model.

To avoid the problems associated with missing values in FIB-4 and Forns indexes, we performed an additional analysis after multiple imputation procedures. Firstly, 20 new datasets were generated by imputing the missing values for each fibrosis score with linear regression, using as covariates age, sex, height and weight. Then, we calculated the HR for each variable of interest by averaging the values obtained for each filled-in data set.

All reported $p$ values are two-tailed. The level of statistical significance was set at $p<0.05$. All the calculations were performed with the software Stata version 14 (StataCorp, 4905 Lakeway Drive, College Station, Texas 77845 USA). Plots were created with R software version 3.6.3. 


\section{Ethical issues}

The study was approved by the local Ethics Committee (May $11^{\text {th }} 2020$ ).

RESULTS

Baseline characteristics of the included patients are shown in Table 1. Two hundred fourteen patients were admitted to the ICU during the study period, 154 of them (71.96\%) men. The mean age at hospital admission was 59.58 (13.79) years. The most frequently observed comorbidities were hypertension and dyslipidaemia (52.8\% and 43.93\%, respectively). Diabetes was present in $21.96 \%$ of the cases, while only $7.48 \%$ of the patients had chronic pulmonary obstructive disease. Sixteen patients $(7.48 \%)$ had a previous diagnosis of chronic liver disease, but only two of them had advanced fibrosis. Specific treatments for COVID-19 are detailed in Table 1.

The median follow-up time (starting at the day of hospital admission) was 38 (IQR 22-54) days. At the end of the study, 84 patients were dead (39.25\%), $69(32.24 \%)$ had been discharged, $34(15.89 \%)$ were alive and transferred to conventional wards and 27 (12.62\%) were still in the ICU.

\section{Liver fibrosis scores}

The Forns and FIB-4 indexes prior to hospital admission could be calculated in $156(72.90 \%)$ and $177(82.71 \%)$ cases, respectively. Mean values for the two scores were 5.37 (1.60) and 1.55 (1.03). Estimation of liver fibrosis 
before COVID-19 admission showed that only $21.15 \%$ of patients according to the Forns index, and $54.24 \%$ according FIB-4, had no or minimal fibrosis (F0F1) (Figure 1A). As expected, those patients in whom liver fibrosis scores were available had a greater proportion of comorbidities. However, previously diagnosed liver disease had a similar frequency in both groups (Supplementary table 1).

Since NFS was available only in $44 \%$ of the cases (mainly due to the lack of albumin value) this index was not considered for statistical comparisons. However, the distribution of the available NFS values was consistent with the fibrosis categories determined by baseline Forns or FIB-4 (Figure 1B).

\section{Baseline factors related to survival}

As shown in Table 2, age, history of past or active smoking and different comorbidities (hypertension, diabetes, dyslipidaemia, cardiovascular disease, COPD and chronic kidney disease) were associated with death. The $\mathrm{CCl}$ was also significantly higher in patients who died. Treatment with angiotensin converting enzyme inhibitors (ACEI) or angiotensin receptor blockers (ARB) was related to death in the overall cohort (38 [45.24\%] vs. $39[30 \%], p=0.023$ ) but not in the subgroup of patients with hypertension (37 [66.07\%] vs. 38 [66.67\%], $p=0.947)$. Importantly, known chronic liver disease was not associated with worse outcomes.

Both liver fibrosis scores calculated before COVID-19 admission were significantly greater in non-survivors than in survivors (Table 2). Moreover, the 
probability of survival was significantly lower in patients who had any degree of fibrosis estimated either by Forns or FIB-4 indexes. Additionally, mortality increased according to the severity of fibrosis estimated by the Forns index and by FIB-4 (Figure 2; $p$ value for trend $<0.05$ for both indexes). The mortality rate of patients in which liver fibrosis indexes could not be calculated was similar to that in the rest of the cohort (Supplementary table 1).

Figure 3 depicts the comparison of laboratory values between survivors and non-survivors at different time points. As shown, there were no relevant differences in ALT, AST or bilirubin levels between the two groups at hospitalization, at the time of ICU admission or considering the highest value of each parameter during evolution.

\section{Multivariate analysis}

In univariate analysis, history of previous or active smoking, $\mathrm{CCl}$, creatinine, serum albumin, APACHE II, serum ferritin and both fibrosis indexes were associated with mortality. Interestingly, Forns index and FIB-4 were independently associated with survival after adjustment for $\mathrm{CCI}$, APACHE II and serum ferritin (Table 3).

These results were reproduced when a multiple imputation method was used for the management of Forns and FIB-4 missing values (Supplementary table 2). 
Several studies have suggested that alterations in liver function tests may play a relevant role in the natural history of COVID-19 [1,4,8,9,19]. However, the contribution of pre-established liver disease to the prognosis of COVID-19 has not been fully assessed. Importantly, the vast majority of available information has evaluated the influence on different outcomes of altered liver function tests when COVID-19 is already established, and it is difficult to ascertain whether they represent real markers of pre-existent liver disease or they are manifestations of SARS-CoV-2 infection. Here, we describe that increased values of liver fibrosis scores commonly used in clinical practice (FIB-4 and Forns indexes) obtained before COVID-19 initiation, and therefore indicative of a possible pre-existing chronic liver disease, are related to survival of ICU hospitalized patients with COVID-19. Moreover, this association remains significant after adjustment for two main components of prognosis in ICU patients: chronic comorbidity (estimated in our study by the Charlson Comorbidity Index) and severity of the clinical condition at the time of ICU admission (evaluated by the APACHE II) and also for the intensity of the inflammatory response (estimated by serum ferritin values). Importantly, the Charlson index has been identified as a robust predictor of mortality in ICU patients with acute lung injury and severe acute respiratory distress syndrome (ARDS), independently of its aetiology $[20,21]$ and has been associated with poorer outcomes in chronic liver disease [22]; furthermore, the APACHE II is a widely validated score for the assessment of prognosis in critically ill patients, including those with acute respiratory failure and ARDS as a primary cause of admission $[23,24]$. Our findings confirm a recent report indicating that the 
severity of COVID-19 was significantly less intense in patients with a low risk of the fibrosis FIB-4 category as compared with those with intermediate or high fibrosis categories [8]. One advantage of our study is that it is focused on the most severe patients (i.e. patients admitted to the ICU). Although the selection of this population increases the statistical power of the analysis due to the number of events, it also limits the generalization of our results to other relevant less severe settings. However, a recent multicentre study including a large population of unselected in-hospital Spanish patients identified cirrhosis as an independent predictive factor, indicating that advanced fibrosis could be a relevant player in COVID-19 prognosis [25]. Another strength of our study is that it is based on calculations of indexes before SARS-Cov-2 infection, without possible interferences in liver test changes intrinsically associated with COVID19. Although it was expected that this would generate a large amount of missing data, we chose this approach in order to avoid the potential bias if we calculated them once the disease was initiated (acute-phase platelet rise, hepatocellular liver injury patterns often normalize AST/ALT ratio to the mean). Importantly, our results were similar after handling missing data by multiple imputation procedures.

It could be argued that the association between these indexes and mortality does not really reflect the presence or the influence of pre-existent liver disease on the prognosis. Much the data from our study contradict this possibility. Firstly, the relatively high prevalence of pre-existent fibrosis estimated in the study is not surprising when considering the high representation of metabolic risk factors present in our population; in fact, the estimated fibrosis rate is similar to that reported in studies examining similar 
populations $[26,27]$. Secondly, both indexes are similarity related to survival, also indicating the consistency of our results; in fact, the mortality rate of patients in which the Forns index or FIB-4 estimated an intermediate or high risk of fibrosis was very similar ( $46 \%$ and $50 \%$, respectively); furthermore, although it was only available in less than half of the cases, NFS also differentiates two populations with a clearly different risk of death $(29 \%$ in the group with presumably healthy livers [cut-off point of $<-1.455$ ] vs. $48 \%$ in the remaining patients, data not shown). Finally, we found that the mortality risk parallels the fibrosis estimation obtained by the three categories of Forns index or FIB-4, suggesting a biologically plausible association. On the other hand, it is also widely recognized that serological indexes predict with reasonable accuracy the stage of liver fibrosis, indicating that they are useful screening tools in clinical practice [14]. The described association between pre-existent fibrosis and the risk of death in COVID-19 patients should not be undervalued, because there are many pieces of evidence emphasizing the prognostic relevance of liver fibrosis in many situations. Similarly, different population-based studies have shown that pathological scores of FIB-4, Forns and NFS are associated with an increased risk of liver-related mortality [16,28]. Finally, our data are consistent with the observations of recent studies showing worse progression of COVID-19 in patients with previously known cirrhosis [4-6] or in those with high FIB-4 values $(9,10)$.

Abnormal serum liver enzymes are frequent in COVID-19, especially in severely ill patients [29]. However, the existence of an association between such alterations and mortality is not well documented. While the impairment of liver tests (aminotransferases, bilirubin) has sometimes been suggested as a 
risk factor of death [19], other studies yielded different results [30]. Therefore, we have also explored the possible influence of liver test evolution during the disease course on survival. Our study suggests that in our population of critically ill patients, liver test impairment throughout COVID-19 progression is mild and not significantly associated with mortality. This is in accordance with the hypothesis that an uncontrolled production of pro-inflammatory mediators leads to the development of ARDS and cytokine storm syndrome, probably responsible for the majority of alterations in liver biochemistry [30]. Moreover, our findings emphasize that the evaluation of liver involvement in COVID-19 patients should rely on the estimation of pre-existent liver disease with noninvasive indexes rather than on the analysis of isolated values of liver tests.

Our study has some limitations. Firstly, in the context of the COVID-19 pandemic, a significant number of patients with severe COVID-19 did not have access to the ICU mainly because of their age and the existence of comorbidities. Consequently, there could be a potential bias because patients at an older age or with greater comorbidity (especially advanced liver disease) would be underrepresented in our series. However, our study was mainly aimed at analysing the influence of previously unknown liver disease on the evolution of severe COVID-19 because the prognostic impact of cirrhosis in patients with different severe acute diseases is well known. Secondly, we were not able to validate our data in an independent series of patients. In this regard, it is noteworthy that our study included a very large cohort of unselected patients consecutively admitted to the ICU at our hospital during the first two months of the COVID-19 pandemic, supporting the validity of our conclusions. On the other hand, our aim was to develop an explicative model to assess the influence 
of pre-existing liver disease on the prognosis of these patients, not to develop a prognostic model that necessarily needs to be validated. Thirdly, we lack additional data to more accurately determine the severity of liver damage or to evaluate the aetiology of the liver disease. Nevertheless, in the epidemiological context of the overwhelming COVID-19 pandemic, the assessment of underlying liver disease, either by non-invasive methods such as liver stiffness measurement or ultrasonography or by invasive procedures such as liver biopsy, was practically impossible. It is noteworthy that there is a discrepancy in the proportion of patients with an estimation of non-significant liver fibrosis between FIB-4 and Forns indexes. Nonetheless, the accuracy of an individual outcome prediction with a particular index did not affect the validity of our overall explicative model. Finally, data regarding the association between fibrosis scores and the outcomes of the overall population of patients admitted with COVID-19 and not only ICU cases are not available.

In conclusion, pre-existing liver fibrosis, as estimated by serological noninvasive measurements, is independently associated with a significantly higher risk of death in patients with severe COVID-19 admitted to the ICU. 
Conflict of interest: the authors declare that they have no conflict of interest.

\section{REFERENCES}

1. Guan W, Ni Z, Hu Y, Liang W, Ou C, He J, et al. Clinical characteristics of coronavirus disease 2019 in China. N Engl J Med. 2020;382:1708-20.

2. Characteristics of SARS-CoV-2 patients dying in Italy. Report based on available data on April 29th, 2020. Epidemiology for public health, Istituto Superiore di Sanità. Available from:

https://www.epicentro.iss.it/en/coronavirus/sars-cov-2-analysis-of-dea.

3. Gacouin A, Locufier M, Uhel F, Letheulle J, Bouju P, Fillatre P, et al. Liver cirrhosis is independently associated with 90-day mortality in Ards patients. Shock. 2016;45:16-21. 
4. Qi X, Liu Y, Wang J, Fallowfield JA, Wang J, Li X, et al. Clinical course and risk factors for mortality of COVID-19 patients with pre - existing cirrhosis : a multicentre cohort study. Gut (In press).

5. Singh S, Khan A. Clinical Characteristics and outcomes of COVID-19 among patients with pre-existing liver disease in United States: a multi-center research network study. Gastroenterology 2020;159:768-771

6. lavarone $\mathrm{M}$, Ambrosio RD, Soria $\mathrm{A}$, et al. High rates of 30-day mortality in patients with cirrhosis and COVID-19. J Hepatol 2020;S0168-8278(20)30365-2. 7. Ji D, Qin E, Xu J, Zhang D, Cheng G, Wang Y, et al. Non-alcoholic fatty liver diseases in patients with COVID-19: a retrospective study. J Hepatol. 2020;73:451-453.

8. Targher G, Mantovani A, Byrne CD, Wang X-B, Yan H-D, Sun Q-F, et al. Risk of severe illness from COVID-19 in patients with metabolic dysfunctionassociated fatty liver disease and increased fibrosis scores. Gut. 2020; 69:1545-1547.

9. Ibáñez-Samaniego L, Bighelli F, Usón C, et al. Elevation of liver fibrosis index FIB-4 is associated with poor clinical outcomes in patients with COVID-19. J Infect Dis. 2020; 222:726-733.

10. Charlson ME, Pompei P, Ales K, McKenzie C. A new method of classifying prognostic in longitudinal studies : development and validation. J Chronic Dis. 1987;40:373-83.

11. Knaus W, Draper E, Wagner D, Zimmerman J. APACHE II: a severity of disease classification system. Crit Care Med. 1985;13:818-29.

12. Vallet-Pichard A, Mallet V, Nalpas B, Verkarre V, Nalpas A, Dhalluin-Venier V, et al. FIB-4: an inexpensive and accurate marker of fibrosis in HCV infection. 
Comparison with liver biopsy and FibroTest. Hepatology. 2007;46:32-6.

13. Sterling RK, Lissen E, Clumeck N, Sola R, Correa MC, Montaner J, et al. Development of a simple noninvasive index to predict significant fibrosis in patients with HIV/HCV coinfection. Hepatology. 2006;43:1317-25.

14. Vilar-Gomez E, Chalasani N. Non-invasive assessment of non-alcoholic fatty liver disease: clinical prediction rules and blood-based biomarkers. $\mathrm{J}$ Hepatol. 2018;68:305-15.

15. Forns X, Ampurdanes S, Llovet JM, Aponte J, Martı E, Forns X, et al. Identification of chronic hepatitis $\mathrm{C}$ patients without hepatic fibrosis by a simple predictive model. Hepatology. 2002;36:986-92.

16. Nabi O, Lacombe K, Boursier J, Mathurin P, Zins M, Serfaty L. Prevalence and risk factors of nonalcoholic fatty liver disease and advanced fibrosis in general population: the french nationwide NASH-CO study. Gastroenterology 2020;159:791-793.

17. Angulo P, Hui JM, Marchesini G, Bugianesi E, George J, Farrell GC, et al. The NAFLD fibrosis score: a noninvasive system that identifies liver fibrosis in patients with NAFLD. Hepatology. 2007;45:846-54.

18. Bhatraju PK, Ghassemieh BJ, Nichols M, Kim R, Jerome KR, Nalla AK, et al. Covid-19 in critically ill patients in the Seattle Region - case series. N Engl J Med. 2020;382:2012-22.

19. Lei F, Liu YM, Zhou F, Qin JJ, Zhang P, Zhu L, et al. Longitudinal association between markers of liver injury and mortality in COVID-19 in China. Hepatology. 2020; 72:389-398.

20. Seuransky JE, Martin GS, Mendgz-Tellez P, Shanholtz C, Brower R. Pulmonary vs nonpulmonary sepsis and mortality in acute lung injury. Chest. 
2008;134:534-8.

21. Ando K, Doi T, Moody SY, Ohkuni Y. The effect of comorbidity on the prognosis of acute lung injury and acute respiratory distress syndrome. Intern Med. 2012;51:1835-40.

22. Powell EE, Skoien R, Rahman T, Clark PJ, O’Beirne J, Hartel G, et al. Increasing hospitalization rates for cirrhosis: overrepresentation of disadvantaged australians. EClinicalMedicine. 2019;11:44-53.

23. Larsson J, Itenov TS, Bestle MH. Risk prediction models for mortality in patients with ventilator- associated pneumonia: a systematic review and metaanalysis. J Crit Care. 2017;37:112-8.

24. Auriemma CL, Zhuo H, Delucchi K, Deiss T, Liu T, Jauregui A, et al. Acute respiratory distress syndrome - attributable mortality in critically ill patients with sepsis. Intensive Care Med. 2020; 46:1222-1231.

25. Berenguer J, Ryan P, Rodríguez-Baño J, Jarrín I, Carratalà J, Pachón J, et al. Characteristics and predictors of death among 4,035 consecutively hospitalized patients with COVID-19 in Spain. Clin Microbiol Infect [Internet]. 2020;S1198-743X(20)30431-6.

26. Rinella ME. Nonalcoholic fatty liver disease a systematic review. JAMA. 2015;313:2263-73.

27. Miyaaki H, Ichikawa T, Taura N, Miuma S, Shibata H, Isomoto H, et al. Predictive value of the fibrosis scores in patients with chronic hepatitis c associated with liver fibrosis and metabolic syndrome. Intern Med. $2011 ; 50: 1137-41$.

28. Angulo P, Bugianesi E, Bjornsson E. Simple noninvasive systems predict long-term outcomes of patients with nonalcoholic fatty liver disease. 
Gastroenterology. 2013;145:782-9.

29. Bloom PP, Meyerowitz EA, Reinus Z, Daidone M, Gustafson J, Kim AY, et al. Liver biochemistries in hospitalized patients with COVID-19. Hepatology. (In press).

30. Bangash MN, Patel J, Parekh D. COVID-19 and the liver: little cause for concern. Lancet Gastroenterol Hepatol. 2020;5:529-30. 


\begin{tabular}{|c|c|c|}
\hline Age (years) & 59.58 & (13.79) \\
\hline Sex (male) & $154 / 214$ & $(71.96)$ \\
\hline \multicolumn{3}{|l|}{ Race } \\
\hline Caucasian & $161 / 214$ & $(75.23)$ \\
\hline Hispanics & $48 / 214$ & $(22.43)$ \\
\hline Others & $5 / 214$ & $(2.34)$ \\
\hline \multicolumn{3}{|l|}{ Cardiovascular risk factors } \\
\hline Hypertension & $113 / 214$ & $(52.80)$ \\
\hline Treatment with ACEI or ARB & $77 / 214$ & $(35.98)$ \\
\hline Dyslipidaemia & $94 / 214$ & $(43.93)$ \\
\hline Diabetes & $47 / 214$ & $(21.96)$ \\
\hline $\mathrm{BMI}\left(\mathrm{Kg} / \mathrm{m}^{2}\right)$ & 30.88 & $(5.74)$ \\
\hline Harmful Alcohol intake & $21 / 214$ & (9.81) \\
\hline Previous or active smoking & $49 / 214$ & (22.90) \\
\hline \multicolumn{3}{|l|}{ Other comorbidities } \\
\hline COPD & $16 / 214$ & $(7.48)$ \\
\hline Chronic kidney disease & $28 / 214$ & $(13.08)$ \\
\hline Charlson index & 1.90 & $(1.98)$ \\
\hline Known history of liver disease & $16 / 214$ & $(7.48)$ \\
\hline \multicolumn{3}{|l|}{ Etiology } \\
\hline Alcohol & $2 / 16$ & $(12.50)$ \\
\hline Hepatitis C & $2 / 16$ & $(12.50)$ \\
\hline MAFLD & $9 / 16$ & $(56.25)$ \\
\hline Others & $3 / 16$ & (18.75) \\
\hline \multicolumn{3}{|l|}{ Severity of liver disease } \\
\hline Mild fibrosis & $14 / 16$ & $(87.50)$ \\
\hline
\end{tabular}




\begin{tabular}{lll}
\hline cACLD & $1 / 16$ & $(6.25)$ \\
Decompensated liver disease & $1 / 16$ & $(6.25)$ \\
Specific treatment for COVID-19 & & \\
Lopinavir/ritonavir & $207 / 214$ & $(96.73)$ \\
Hydroxycloroquine & $211 / 214$ & $(98.60)$ \\
Remdesivir & $35 / 214$ & $(16.36)$ \\
Beta-interferon & $96 / 214$ & $(44.86)$ \\
Azithromycin & $97 / 214$ & $(45.33)$ \\
Ceftriaxone & $167 / 214$ & $(78.37)$ \\
Corticosteroids & $182 / 214$ & $(85.05)$ \\
Tocilizumab & $166 / 214$ & $(77.57)$ \\
\hline
\end{tabular}

Table 1. Baseline and COVID-19 related characteristics $(\mathrm{N}=214)$. Data are expressed as mean (SD) or $n(\%)$. ACEl: Angiotensin Converting Enzyme Inhibitors; ARB: Angiotensin Receptor Blockers; BMI: body mass index; COPD: chronic obstructive pulmonary disease; MAFLD: metabolic associated liver disease; cACLD: compensated advanced chronic liver disease. 


\begin{tabular}{|c|c|c|c|c|c|}
\hline \multirow[b]{2}{*}{ Age (years) } & \multicolumn{2}{|c|}{$\begin{array}{l}\text { Non-survivors } \\
\qquad(n=84)\end{array}$} & \multicolumn{2}{|c|}{$\begin{array}{l}\text { Survivors } \\
\qquad(n=130)\end{array}$} & \multirow{2}{*}{$\begin{array}{l}\text { p value } \\
<0.001\end{array}$} \\
\hline & 64.36 & $(9.80)$ & 56.49 & $(15.09)$ & \\
\hline Sex (male) & 65 & (77.38) & 89 & $(68.46)$ & 0.156 \\
\hline \multicolumn{6}{|l|}{ Race } \\
\hline - Caucasian & 63 & (75) & 98 & (73.38) & \\
\hline - Hispanics & 19 & $(22.62)$ & 29 & (22.31) & \\
\hline - $\quad$ Others & 2 & $(2.38)$ & 3 & $(2.31)$ & 0.998 \\
\hline Hypertension & 56 & $(66.67)$ & 57 & $(43.85)$ & 0.001 \\
\hline Diabetes & 25 & (29.76) & 22 & (16.97) & 0.027 \\
\hline Dyslipidemia & 44 & $(52.34)$ & 50 & $(38.46)$ & 0.045 \\
\hline $\operatorname{BMI}\left(\mathrm{kg} / \mathrm{m}^{2}\right)$ & 30.79 & $(5.69)$ & 30.95 & $(5.79)$ & 0.844 \\
\hline Active or previous Smoking & 28 & (33.33) & 22 & (16.92) & 0.006 \\
\hline Harmful alcohol intake & 8 & $(9.52)$ & 13 & $(10.08)$ & 0.895 \\
\hline Cardiovascular disease & 55 & $(65.48)$ & 60 & $(46.15)$ & 0.006 \\
\hline COPD & 12 & $(14.29)$ & 4 & $(3.08)$ & 0.002 \\
\hline Chronic kidney disease & 19 & $(22.28)$ & 9 & $(6.92)$ & 0.001 \\
\hline Known chronic liver disease & 8 & $(9.52)$ & 8 & $(6.15)$ & 0.360 \\
\hline Charlson index & 2.59 & $(2.20)$ & 1.46 & $(1.70)$ & $<0.001$ \\
\hline Treatment with ACEI or ARB & 38 & $(45.24)$ & 39 & (30) & 0.023 \\
\hline $\begin{array}{c}\text { Forns index * Continuous score } \\
\text { Forns index Categories }\end{array}$ & 6.04 & $(1.42)$ & 4.99 & $(1.58)$ & $<0.001$ \\
\hline$<4.2$ & 5 & $(8.20)$ & 28 & $(29.47)$ & \\
\hline $4.2-6.9$ & 40 & $(65.57)$ & 59 & $(62.11)$ & \\
\hline$>6.9$ & 16 & $(26.23)$ & 8 & $(8.42)$ & $<0.001$ \\
\hline
\end{tabular}




\begin{tabular}{c|cc|cc|c}
\hline $\begin{array}{c}\text { FIB-4** Continuous score } \\
\text { FIB-4 Categories }\end{array}$ & 1.77 & $(1.17)$ & 1.41 & $(0.91)$ & 0.020 \\
$<1.45$ & 29 & $(41.43)$ & 67 & $(62.62)$ & \\
$1.45-3.25$ & 37 & $(52.86)$ & 37 & $(34.58)$ & \\
$>3.25$ & 4 & $(5.71)$ & 3 & $(2.80)$ & 0.017 \\
& & & & & \\
\hline
\end{tabular}

Table 2: Comparison of Baseline characteristics between survivors and non survivors. Continuous variables are shown as mean (SD) and categorical variables as $n(\%)$. T-Student test was used for comparisons between means and Chi squared or Fisher's exact test for comparison between categorical variables. BMI: body mass index; COPD: chronic obstructive pulmonary disease; ACEI: ACE inhibitors; ARB: angiotensin receptor blockers. ${ }^{*}$ Forns Index was available in 156 patients. ${ }^{* *}$ FIB-4 was available in 177 patients. 


\begin{tabular}{|c|c|c|c|c|c|c|}
\hline & \multicolumn{2}{|l|}{ Univariate } & \multicolumn{2}{|c|}{$\begin{array}{c}\text { Multivariate } \\
\text { (Forns) } \\
(n=110)\end{array}$} & \multicolumn{2}{|c|}{$\begin{array}{c}\text { Multivariate } \\
\begin{array}{c}\text { (FIB-4) } \\
(n=122)\end{array}\end{array}$} \\
\hline Variable & $\mathrm{HR}(95 \% \mathrm{Cl})$ & $\mathbf{p}$ & $\mathrm{HR}(95 \% \mathrm{Cl})$ & $\mathbf{p}$ & $\mathrm{HR}(95 \% \mathrm{Cl})$ & p \\
\hline Sex (male as reference) & $0.75(0.45-1.25)$ & 0.269 & & & & \\
\hline Previous or active smoking & $1.73(1.09-2.74)$ & 0.019 & $1.35(0.67-2.73)$ & 0.403 & $1.29(0.65-2.56)$ & 0.463 \\
\hline Harmful Alcohol intake & $0.93(0.45-1.94)$ & 0.856 & & & & \\
\hline $\mathrm{BMI}\left(\mathrm{kg} / \mathrm{m}^{2}\right)$ & $1.00(0.96-1.04)$ & 0.852 & & & & \\
\hline Charlson & $1.20(1.09-1.32)$ & $<0.001$ & $1.22(1.00-1.48)$ & 0.046 & $1.25(1.08-1.44)$ & 0.003 \\
\hline \multicolumn{7}{|l|}{ Race (caucasian as } \\
\hline Hispanics & $1.17(0.69-1.97)$ & 0.568 & & & & \\
\hline Others & $1.07(0.25-4.56)$ & 0.925 & & & & \\
\hline Platelets $\left(10^{3} / \mathrm{ml}\right)$ & $1.00(0.99-1.00)$ & 0.312 & & & & \\
\hline $\mathrm{ALT}(\mathrm{UI} / \mathrm{L})$ & $1.00(0.99-1.00)$ & 0.356 & & & & \\
\hline AST (UI/L) & $1.00(0.99-1.00)$ & 0.797 & & & & \\
\hline Bilirubin (mg/dl) & $1.08(0.80-1.47)$ & 0.609 & & & & \\
\hline INR & $0.93(0.51-1.72)$ & 0.826 & & & & \\
\hline Creatinine (mg/dl) & $1.37(0.96-1.97)$ & 0.084 & $1.65(0.70-3.90)$ & 0.254 & $1.12(0.59-2.12)$ & 0.737 \\
\hline Albumin (g/dl) & $0.65(0.41-1.04)$ & 0.076 & $0.86(0.37-1.97)$ & 0.714 & $0.85(0.41-1.77)$ & 0.670 \\
\hline Fibrinogen (mg/dl) & $1.00(1.00-1.01)$ & 0.566 & & & & \\
\hline C-Reactive Protein (mg/dl) & $1.01(0.98-1.02)$ & 0.762 & & & & \\
\hline Ferritin $(\mu \mathrm{g} / \mathrm{l})$ & $1.00(1.00-1.00)$ & 0.000 & $1.00(1.00-1.00)$ & 0.027 & $1.00(0.99-1.01)$ & 0.132 \\
\hline APACHE II & $1.06(1.03-1.09)$ & $<0.001$ & $1.05(0.99-1.10)$ & 0.080 & $1.05(1.00-1.10)$ & 0.044 \\
\hline
\end{tabular}




\begin{tabular}{c|cc|cc|cc}
\hline Baseline Forns & $1.40(1.17-1.67)$ & $<0.001$ & $1.41(1.11-1.81)$ & 0.006 & & \\
\hline Baseline FIB-4 & $1.31(1.08-1.60)$ & 0.007 & & & $1.31(0.99-1.72)$ & 0.051 \\
\hline
\end{tabular}

Table 3. Uni and multivariate Cox models with Forns and FIB-4 indexes respectively. Baseline characteristics, laboratory data at ICU admission and APACHE II score were included. Variables with $p<0.10$ in the univariate analysis were included in the multivariate models. AST: aspartate aminotransferase; ALT: alanine aminotransferase; INR: international normalized ratio; BMI: body mass index. 


\section{Figures}
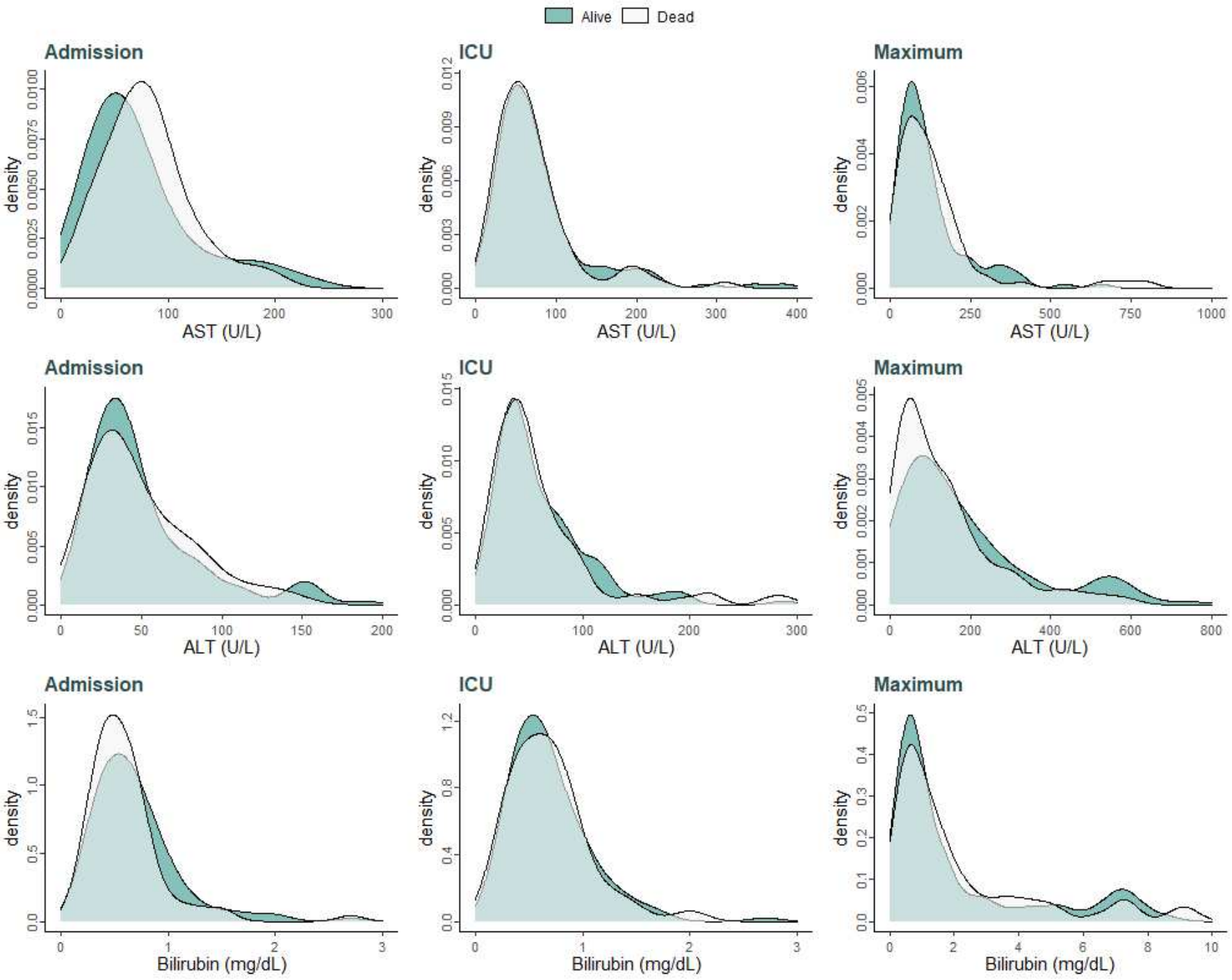

\section{Figure 1}

Panel A Distribution of patients according to different fibrosis categories established by baseline Forns and FIB-4. Panel B Stratification of NAFLD fibrosis score values $(n=95)$ according fibrosis severity determined by Forns and FIB-4. $p$-value for mean comparison between categories $<0.01$ for both indexes. 


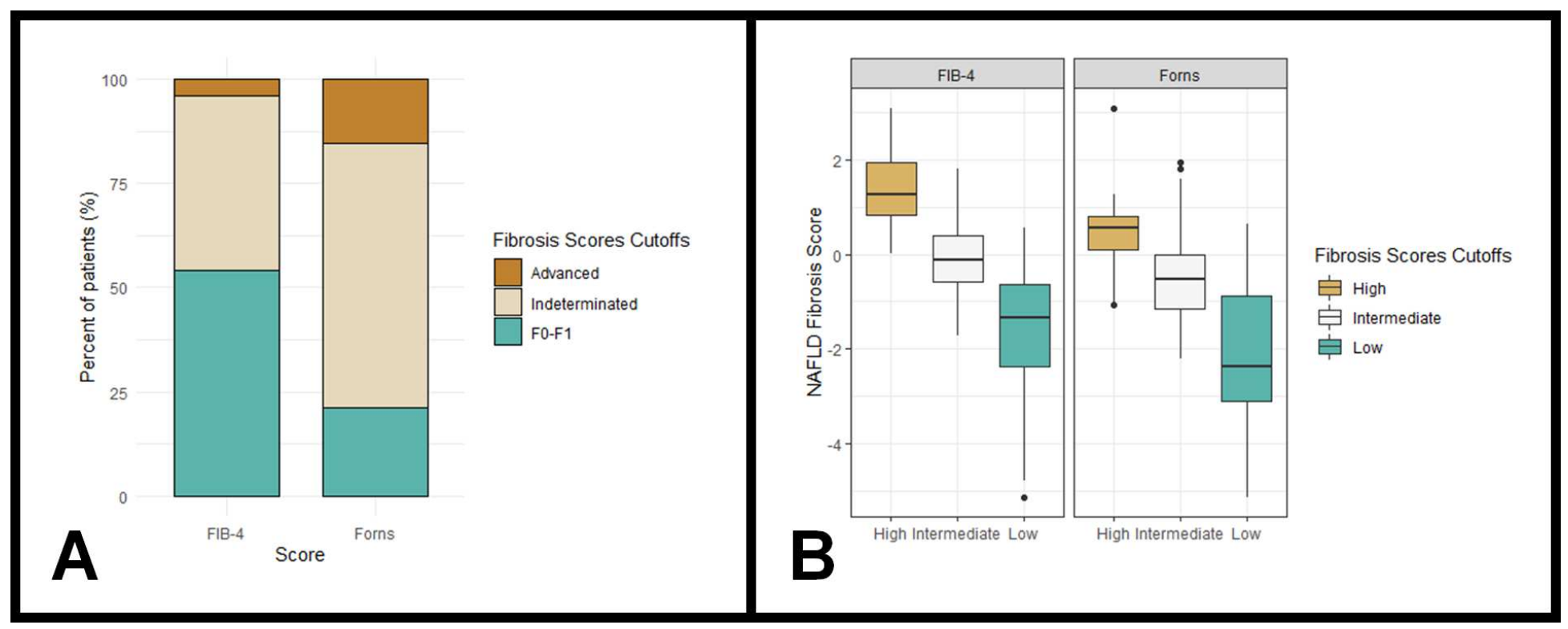

\section{Figure 2}

Kaplan - Meier survival curves according to pre-existing fibrosis. Panel A Survival curves according to the severity of fibrosis estimated by Forns index. Panel B Survival curves according to the presence or absence of fibrosis estimated by Forns Index. Panel C Survival curves according to the severity of fibrosis estimated by FIB-4 index. Panel D Survival curves according to the presence or absence of fibrosis estimated by FIB-4 


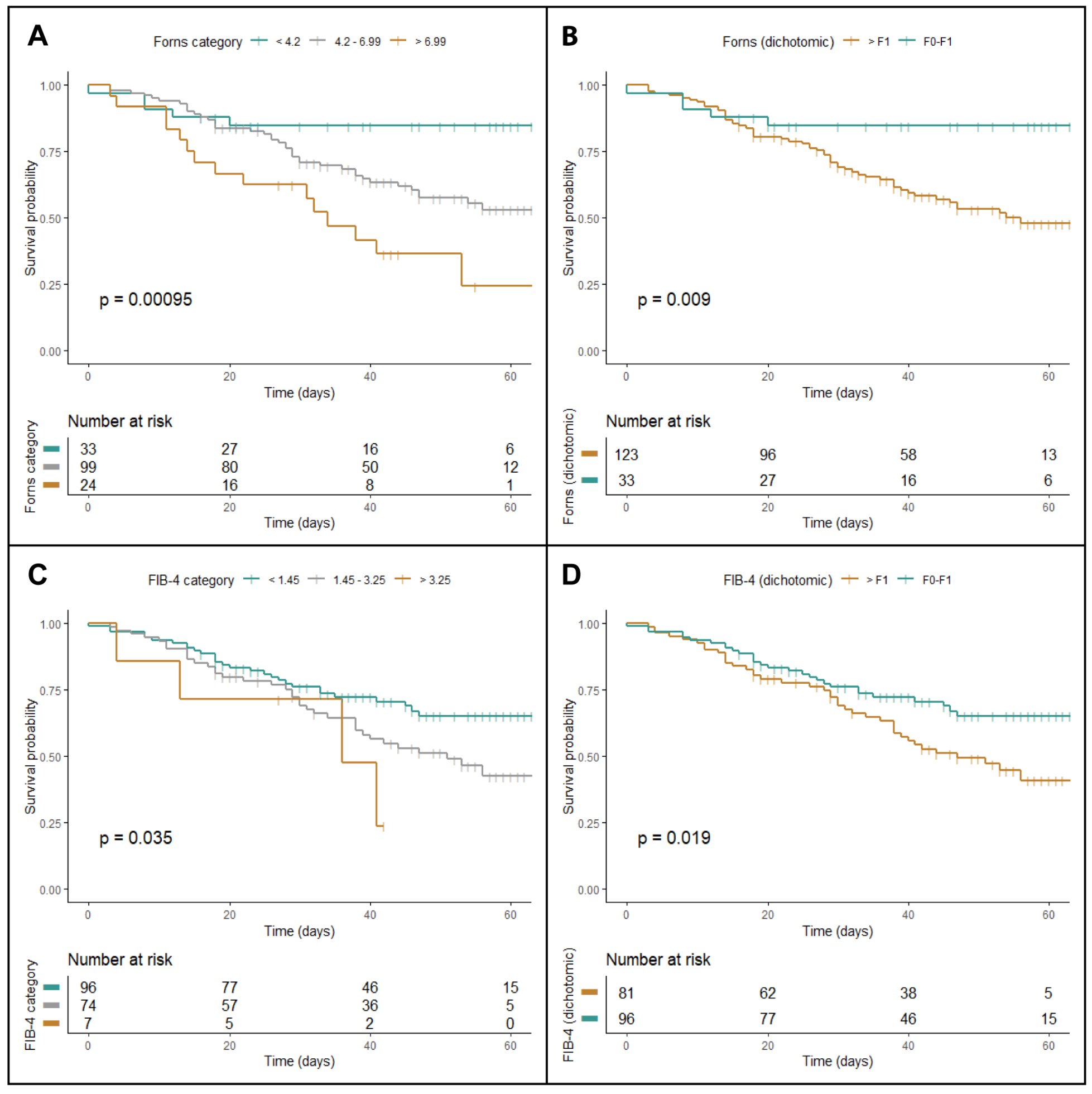

Figure 3

Distribution of different liver laboratory tests in survivors and non-survivors at different time points during hospitalization. Kernel density estimates were used for graphical representation. p-values were nonsignificant in all cases

\section{Supplementary Files}


This is a list of supplementary files associated with this preprint. Click to download.

- Supplementarytable1.docx

- SupplementaryTable2.docx 